PAPER

\title{
Finite-difference time-domain analysis on leak transmission characteristics of narrow gaps
}

\author{
Takumi Asakura $^{1, *}$ and Shinichi Sakamoto ${ }^{2}$ \\ ${ }^{1}$ Institute of Technology, SHIMIZU Corporation, \\ 3-4-17, Etchujima, Koto-ku, Tokyo, 135-8530 Japan \\ ${ }^{2}$ Institute of Industrial Science, the University of Tokyo, \\ 4-6-1, Komaba, Meguro-ku, Tokyo, 153-8505 Japan
}

(Received 26 August 2010, Accepted for publication 8 March 2011)

\begin{abstract}
The sound insulation performance of such wall systems as window sashes, doors, and movable partition walls is often affected by sound waves propagating through narrow gaps that exist at their peripheral parts. In this paper, the leak transmission characteristics through narrow gaps existing in the window sash was investigated through numerical and experimental studies. First, the validity of the finite-difference time-domain (FDTD) method with nonuniform-mesh system was confirmed through 1-dimensional and 2-dimensional numerical studies. Second, narrow gaps at the peripheral parts of a window sash were numerically modeled and their leak transmission characteristics were calculated by the 3-dimensional FDTD method. To evaluate the calculation results, they were compared with measurement results of leak transmission characteristics of a real window sash. As a result, numerical results showed good agreement with the experimental results.
\end{abstract}

Keywords: Leak transmission, Gap, Finite-difference time-domain method, Nonuniform-mesh

PACS number: 43.55.Rg, 43.55.Ka [doi:10.1250/ast.32.182]

\section{INTRODUCTION}

The sound environment inside residential buildings is influenced by sound transmitted through wall systems. The sound insulation performance of wall systems, comprising, for example, window sashes, doors, and movable partition walls, which are installed in the building facade or in the interior space, is often affected by leak transmission through narrow gaps at their peripheral parts.

The sound transmission through a circular or slitshaped aperture has been theoretically investigated [1-5]. More practical case studies on sound insulation performance of real building elements with peripheral gaps, such as doors and windows have also been investigated theoretically and experimentally [6-9]. The sound insulation performance of narrow gaps around window sash have been theoretically investigated by Lewis [6] and Burgess [7]. Burgess applied Helmholtz resonance theory to predict the sound insulation performance of the gaps. Hongisto and co-workers applied Gomperts' theory [4] to predict the sound insulation performance of doors consisting of door panels and peripheral gaps [8,9]. Althogh these

*e-mail: t_asakura@shimz.co.jp theoretical methods are easily applicable to the calculation of the sound insulation performance of gaps with simple cross sections, it is difficult to predict the sound insulation performance of gaps with more complex cross sections. In order to simulate the sound insulation of a gap with a complex structure, wave-based numerical methods are applicable. As a numerical method, the finite element method (FEM), boundary element method (BEM) or finitedifference time-domain (FDTD) method is often used in architectural acoustics. Among those methods, FDTD enables us to calculate directly the transient acoustic propagation using a computer with a relatively small memory capacity. In addition, we can also easily obtain the frequency response by using FFT. We have recently been applying FDTD analysis to problems regarding sound insulation of materials such as glass plates and plasterboard [10]. When we treat sound insulation of more complex wall systems such as window sashes, doors, and movable partitions, narrow gaps might occur in the peripheral parts of the walls and sound transmission through such gaps has severe effects on the total sound insulation of the elements. For this reason, the sound transmission through narrow gaps existing in the peripheral parts of the elements should be modeled and taken into consideration in the analysis. In 
such a case, FDTD with a nonuniform-mesh system should be used in order to model the narrow gaps. In this simulation, the sound field only around the gap structure is discretized with fine meshes and the other field is discretized with relatively coarse meshes. The application of this numerical scheme contributes to the reduction of required memory size and computational time. An investigation on the nonuniform-mesh FDTD in electromagnetics for the antenna design and optimization was conducted by Jiang and Arai [11] and a numerical study was performed in order to reduce the numerical error which arises at the discontinued junction between fine and coarse meshes. On the other hand, in architectural acoustics, there has been no research on the nonuniform-mesh FDTD for acoustical analysis.

In this paper, the applicability of the nonuniform-mesh FDTD analysis for the simulation of sound transmission through narrow gaps is discussed. First, the validity of the nonuniform-mesh FDTD simulation is investigated through 1-dimensional and 2-dimensional numerical study. Then, narrow gaps in the peripheral parts of a window sash were numerically modeled and their leak transmission characteristics were calculated by the 3-dimensional FDTD method with the nonuniform-mesh system. Lastly, the numerical results were compared with measurement results [12] of the sound energy transmitted through narrow gaps in the peripheral parts of a real window sash.

\section{FDTD ANALYSIS WITH NON-UNIFORM MESH SYSTEM}

The FDTD method with the nonuniform-mesh system was applied in order to calculate the leak transmission through narrow gaps, as shown in Fig. 1(a). Althogh the use of a subgridding scheme, as shown in Fig. 1(b) is also applicable, a resolution technique that can completely avoid numerical divergence in the subgridding scheme has not yet been reported [13]. For this reason, the nonuniformmesh system is applied in this research.

When FDTD method with non-uniform mesh system is applied, numerical reflection error arising at the boundary between different-sized meshes might be a problem. This error is considered to be caused by the difference in numerical velocity, which varies depending on the CFL number [14]. In this chapter, the basic theory of the nonuniform-mesh FDTD is described and the numerical error is evaluated through numerical study.

\subsection{Theory}

A sound wave in a 1-dimensional sound field is expressed as

$$
\frac{\partial p}{\partial t}+\rho c^{2} \frac{\partial u}{\partial x}=0
$$

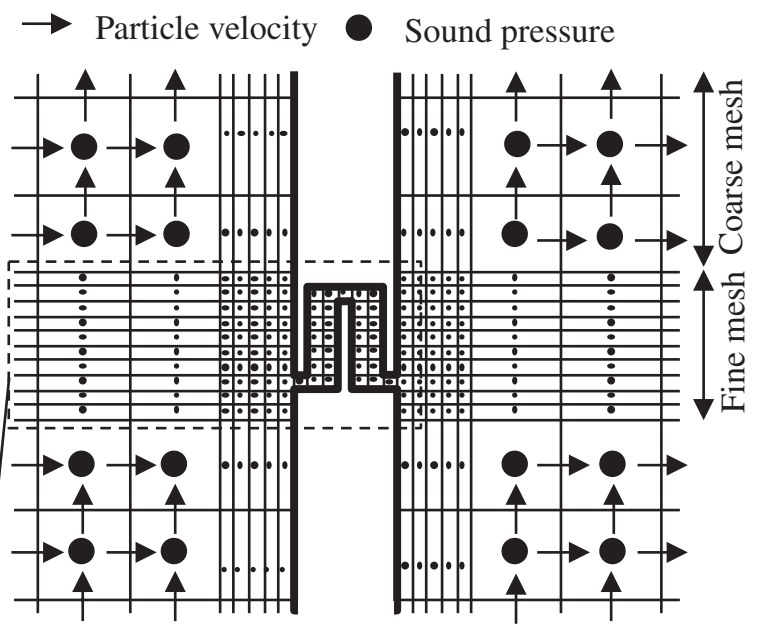

(a) Modeling of the gap by nonuniform-mesh system

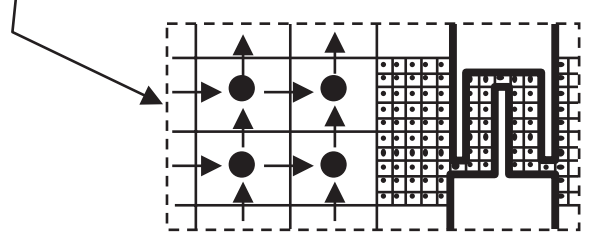

(b) Modeling of the gap by subgridding scheme

Fig. 1 Two-dimensional sound field discretized by nonuniform-mesh system.

$$
\frac{\partial p}{\partial x}+\rho \frac{\partial u}{\partial t}=0
$$

where $p$ is the sound pressure, $u$ is the particle velocity, $\rho$ is the density of air and $c$ is the speed of sound. In FDTD analysis, these equations are transformed into a discrete form in a staggered mesh system. The time and space differential terms in these equations are approximated by a center difference method and expressed as

$$
\begin{aligned}
p_{i}^{n+1 / 2} & =p_{i}^{n-1 / 2}-\rho c^{2} \Delta t \frac{u_{i+1 / 2}^{n}-u_{i-1 / 2}^{n}}{\Delta x} \\
u_{i+1 / 2}^{n+1} & =u_{i+1 / 2}^{n}-\frac{\Delta t}{\rho} \frac{\left(p_{i+1}^{n+1 / 2}-p_{i}^{n+1 / 2}\right)}{\Delta x},
\end{aligned}
$$

where $n$ and $i$ denote the time step and the spatial grid number of the mesh in the $x$-direction, and $\Delta t$ and $\Delta x$ are the time and spatial intervals, respectively. In FDTD analysis using a uniform mesh system, the sound pressure and particle velocity are arranged at regular intervals, as shown in Fig. 2(a), and $u_{i}^{n+1}$ is calculated using $p_{i+1 / 2}^{n+1 / 2}$ and $p_{i-1 / 2}^{n+1 / 2}$, which are arranged at the same distance from $u_{i}^{n+1}$. Figure 2(b) shows the 1-dimensional sound field discretized using the nonuniform-mesh system. In this figure, the ratio of the mesh sizes of fine and coarse meshes is 1:3. The particle velocity, $u_{i_{\mathrm{b}}}^{n+1}$, defined at the boundary between the coarse and fine meshes is calculated using the following equations. In these equations, pressure gradient, $\alpha$, at the boundary is calculated by linear interpolation of sound pressures at 2 points: $p_{i_{\mathrm{b}}+1 / 2}^{n+1 / 2}$ and $p_{i_{\mathrm{b}}-1 / 2}^{n+1 / 2}($ Method A). 
(a) Uniform-mesh system

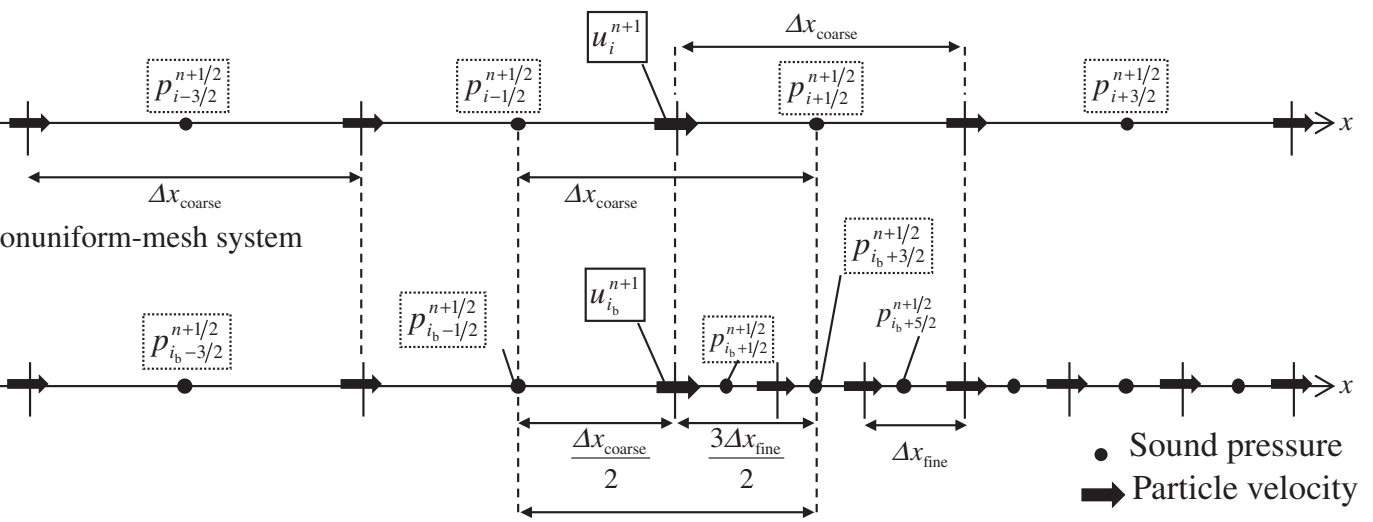

Fig. 2 Arrangement of sound pressure and particle velocity in uniform- and nonuniform-mesh system.

$$
\begin{aligned}
& u_{i_{\mathrm{b}}}^{n+1}=u_{i_{\mathrm{b}}}^{n}-\frac{\Delta t}{\rho} \alpha \\
& \alpha=\frac{p_{i_{\mathrm{b}}+1 / 2}^{n+1 / 2}-p_{i_{\mathrm{b}}-1 / 2}^{n+1 / 2}}{0.5 \cdot\left(\Delta x_{\text {coarse }}+\Delta x_{\text {fine }}\right)}
\end{aligned}
$$

The gradient can also be calculated by polynomial interpolation of sound pressures at 4 points: $p_{i_{\mathrm{b}}-3 / 2}^{n+1 / 2}$, $p_{i_{\mathrm{b}}-1 / 2}^{n+1 / 2}, p_{i_{\mathrm{b}}+1 / 2}^{n+1 / 2}$, and $p_{i_{\mathrm{b}}+3 / 2}^{n+1 / 2}$ (Method B). The calculation method of this gradient, $\alpha$, is described in Appendix. In these methods A and B, particle velocity at the boundary is calculated using the sound pressures at two or four points described above, which are defined at the asymmetrical positions across the boundary, and the center difference method is disregarded only at this boundary. This irregular treatment for updating the particle velocity at the boundary might increase the numerical reflection error. In order to avoid the increase in the numerical error, the following Method C is adopted.

In order to keep the center difference method at the boundary, the particle velocity, $u_{i_{\mathrm{b}}}^{n+1}$, at the boundary is calculated as

$$
u_{i_{\mathrm{b}}}^{n+1}=u_{i_{\mathrm{b}}}^{n}-\frac{\Delta t}{\rho \Delta x_{\text {coarse }}}\left(p_{i_{\mathrm{b}}+3 / 2}^{n+1 / 2}-p_{i_{\mathrm{b}}-1 / 2}^{n+1 / 2}\right)
$$

In this equation, $p_{i_{\mathrm{b}}-1 / 2}^{n+1 / 2}$ and $p_{i_{\mathrm{b}}+3 / 2}^{n+1 / 2}$ are defined at symmetrical positions across the boundary in Fig. 2(b). Although Eq. (7) is specialized for the situation with the mesh ratio $1: 3$, this method is also applicable for any situation in which the ratio between fine and coarse mesh sizes is 1 :(odd number). When the mesh ratio is $1: L$, the particle velocity at the boundary can be calculated by

$$
u_{i_{\mathrm{b}}}^{n+1}=u_{i_{\mathrm{b}}}^{n}-\frac{\Delta t}{\rho \Delta x_{\text {coarse }}}\left(p_{i_{\mathrm{b}}+L / 2}^{n+1 / 2}-p_{i_{\mathrm{b}}-1 / 2}^{n+1 / 2}\right) .
$$

This method was originally applied to FDTD with a subgridding scheme in electromagnetic simulation [15] in order to reduce the numerical error at the boundary between the subgrid area and the coarse grid area.
Considering the stability condition for the calculation of sound pressure and particle velocity in the fine mesh area, a shorter time interval than that for the coarse mesh area is necessary, which leads to an increase in the total calculation time. In order to reduce the calculation time, a locally shorter time interval for the fine mesh area than that for the coarse mesh area is applied. In order to satisfy the locally shorter time interval, iterative calculation for the fine mesh area is performed. The flowchart for the nonuniform-mesh FDTD, including an iterative scheme for the fine mesh area (A), is shown in Fig. 3. FDTD calculation for the whole area is iterated $K$ times. The calculation loop for the fine mesh area is inserted between the calculation of particle velocity and the setting of the boundary condition for the coarse mesh area, and the sound pressure and particle velocity in the fine mesh area, indicated by gray points and arrows in Fig. 4, are updated $M$ times, while those in the coarse mesh area are updated once. Another scheme, in which $p_{i_{\mathrm{b}}-1 / 2}$ is obtained through an interpolation in time [11], can also be implemented however, in this research, such a scheme was not adopted for simplicity of calculation.

In this iterative calculation loop, the iteration number, $M$, the time interval for the fine mesh area, $\Delta t_{\text {fine, and }}$ that for the coarse mesh area, $\Delta t_{\text {coarse, }}$ are set as follows.

The time intervals, $\Delta t_{\text {fine }}$ and $\Delta t_{\text {coarse }}$, are given by the following equations that describe the stability condition for 1-dimensional FDTD analysis.

$$
\begin{aligned}
& \Delta t_{\text {fine }} \leq \frac{\Delta x_{\text {fine }}}{c} \\
& \Delta t_{\text {coarse }} \leq \frac{\Delta x_{\text {coarse }}}{c}
\end{aligned}
$$

$\Delta t_{\text {fine }}$ and $\Delta t_{\text {coarse }}$ are determined from Eqs. (9) and (10) so that the iteration number, $M$, is obtained as an integer number by 


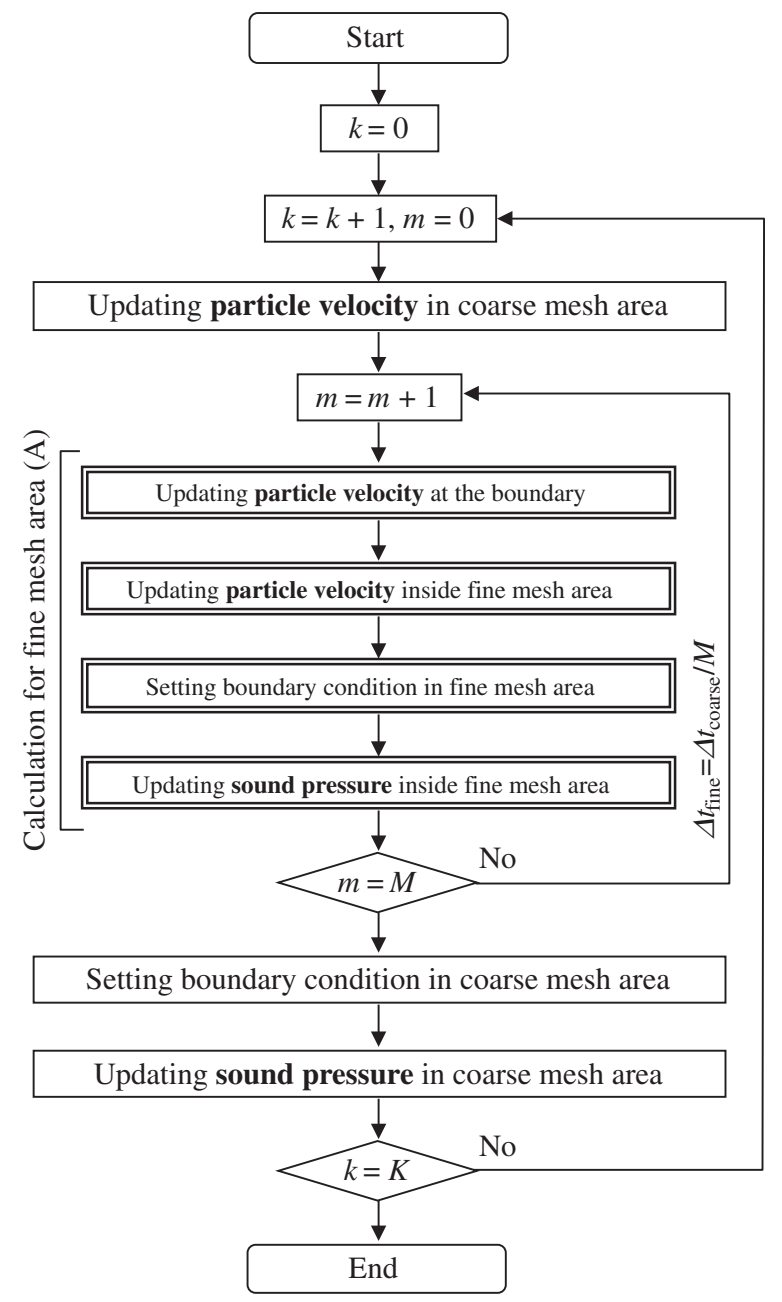

Fig. 3 Flowchart for the nonuniform-mesh FDTD scheme.

$$
M=\frac{\Delta t_{\text {coarse }}}{\Delta t_{\text {fine }}} .
$$

\subsection{Validation of FDTD Method with Nonuniform- Mesh System}

In order to investigate the numerical reflection error caused at the boundary between different-sized meshes, FDTD analyses for 1-dimensional and 2-dimensional sound fields as shown in Figs. 5 and 6, were performed. Details of the parametrical study are presented hereafter.

(1) Study of 1-dimensional sound field

Study 1: Comparison of Methods A, B, and C

The reflection error caused by nonuniform-mesh FDTD using Methods A, B, and C was calculated and compared with each other. The ratio of mesh sizes between fine and coarse meshes was set at 1:21 and the iterative calculation for the fine mesh area was not performed. In the 1-dimensional sound field, a sound source point and a receiving point are set as shown in Fig. 5. As a sound source, the initial sound pressure distribution with the Gaussian profile [16], which includes frequency components up to $1 \mathrm{kHz}$, was adopted. In order to avoid sound reflection at each boundary of the simulated sound field, the characteristic impedance of air $\left(409.7 \mathrm{Ns} / \mathrm{m}^{3}\right)$ was assigned as the normal acoustic impedance of each boundary [17]. Then, the transient response at the receiving point shown in Fig. 5 was calculated. The coarse mesh size, $\Delta x_{\text {coarse }}$, was set to be $4 \mathrm{~cm}$ and the fine mesh size, $\Delta x_{\text {fine }}$, was $0.19 \mathrm{~cm}$ $(=4 \mathrm{~cm} / 21)$ in this case. The time intervals, $\Delta t_{\text {fine }}$ and $\Delta t_{\text {coarse }}$, were set to be $5.0 \times 10^{-6} \mathrm{~s}$, which fulfills the stability condition for the fine mesh area. The numerical scheme for the boundary part was varied as Method A, B, and C. Each condition is named Type A-1, Type B-1, and Type C-1, respectively.

An example of the transient response calculated by Method C ( $\Delta x_{\text {coarse }}$ is $4 \mathrm{~cm}$, mesh ratio is $1: 21$ and there is no iteration) is shown in Fig. 7(a). The reflection sound

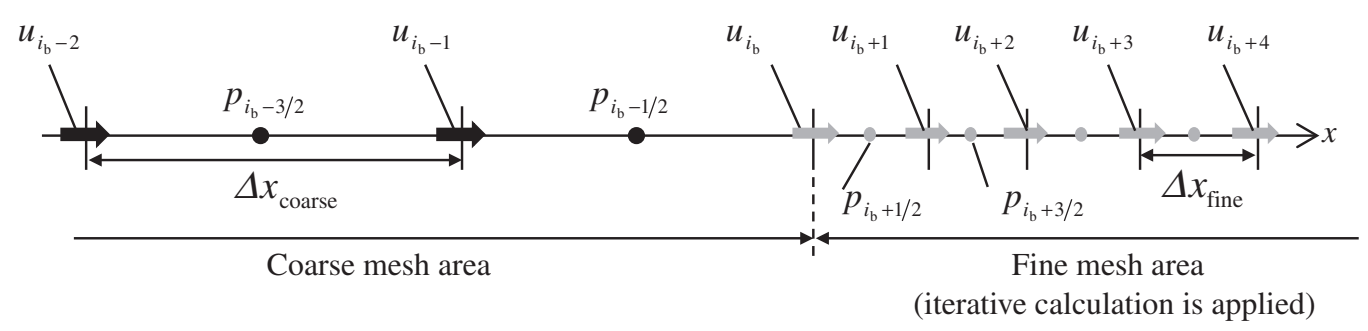

Fig. 4 One-dimensional sound field analysis with iterative calculation for fine mesh area.

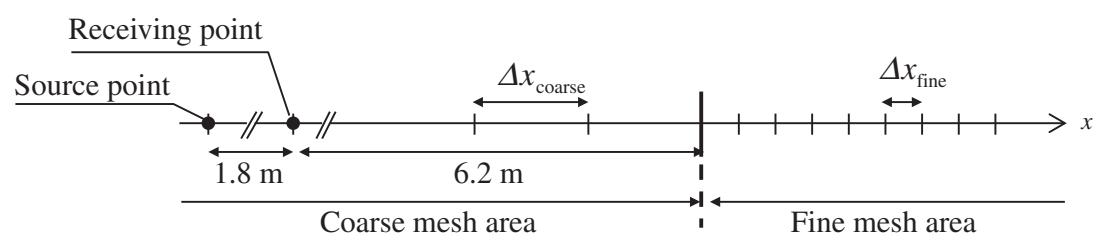

Fig. 5 One-dimensional sound field discretized with nonuniform-mesh system. 


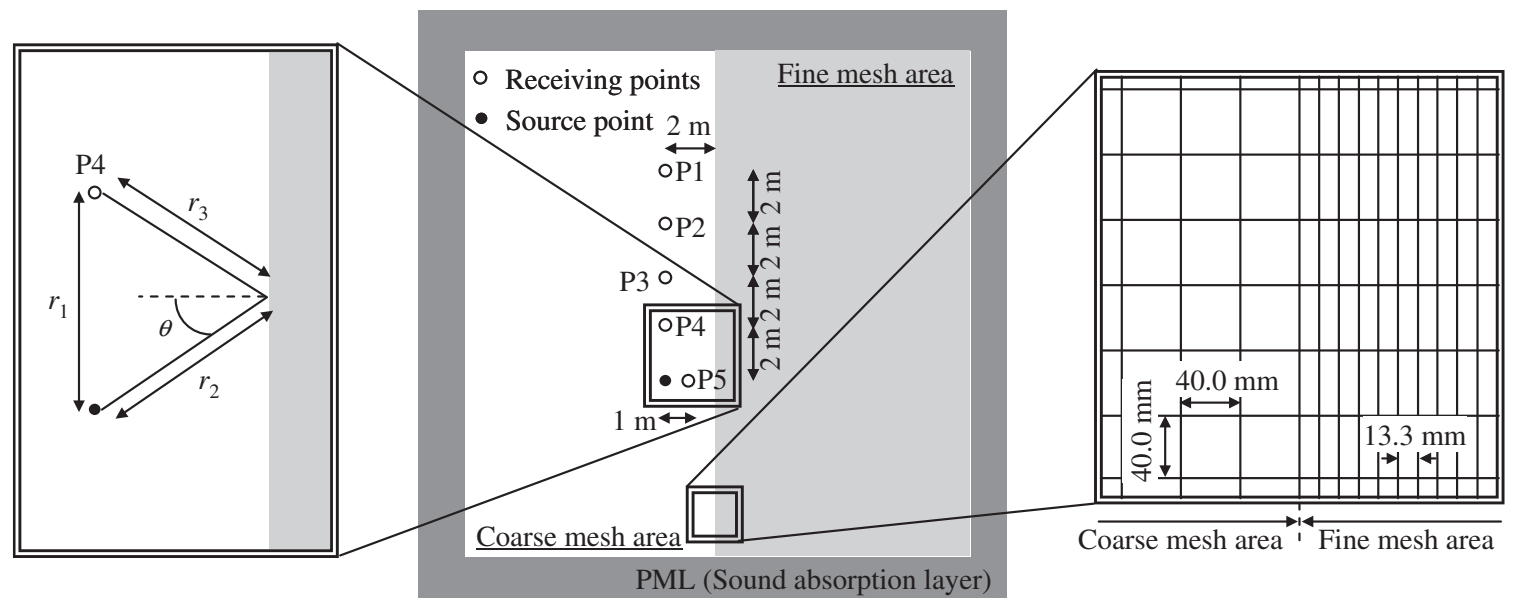

Fig. 6 Two-dimensional sound field discretized with nonuniform-mesh system.

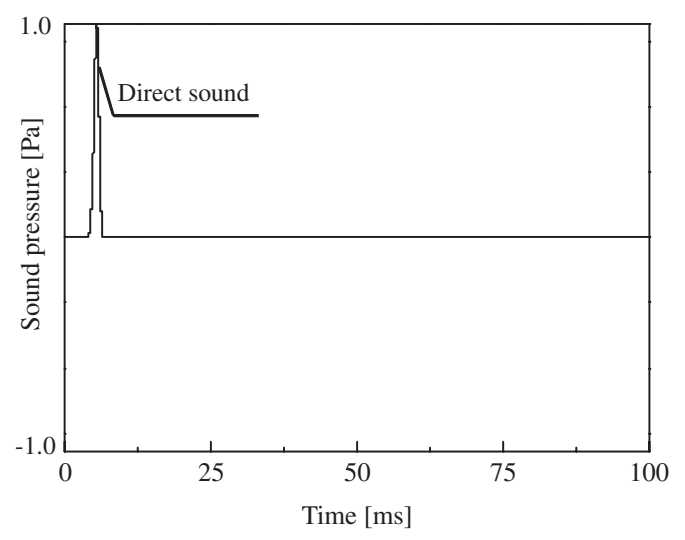

(a) Calculated transient response

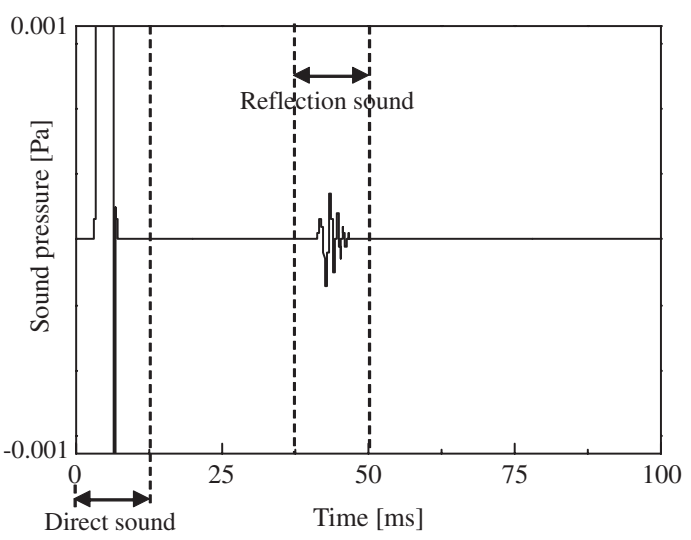

(b) Scaled up figure of the same response

Fig. 7 Example of calculated impulse response.

wave from the boundary is not observed in the figure. In Fig. 7(b), the same transient response is shown with the range of the vertical axis scaled up to 0.001 [Pa]. Each of the direct sound and reflection sound is picked up from the transient response separately, and these responses are transformed into frequency responses using FFT. Finally, the reflection error was calculated as the sound level difference of the magnitudes between these frequency responses.

The calculated reflection errors are shown in Fig. 8. Results for Type B-1 and Type C-1 have almost the same values and Type A-1 yields higher error in all frequency ranges than those of Type B-1 and Type C-1. This indicates that Methods $\mathrm{B}$ and $\mathrm{C}$ are effective for reducing the reflection error caused by the discontinuity of the mesh size.

Study 2: Influence of mesh ratio

The mesh ratio was varied as 1:3 and 1:21, for Methods $\mathrm{B}$ and $\mathrm{C}$. The coarse mesh size, $\Delta x_{\text {coarse }}$, is set as $4 \mathrm{~cm}$ and the fine mesh size, $\Delta x_{\text {fine }}$, is $1.33 \mathrm{~cm}(=4 \mathrm{~cm} / 3)$ and

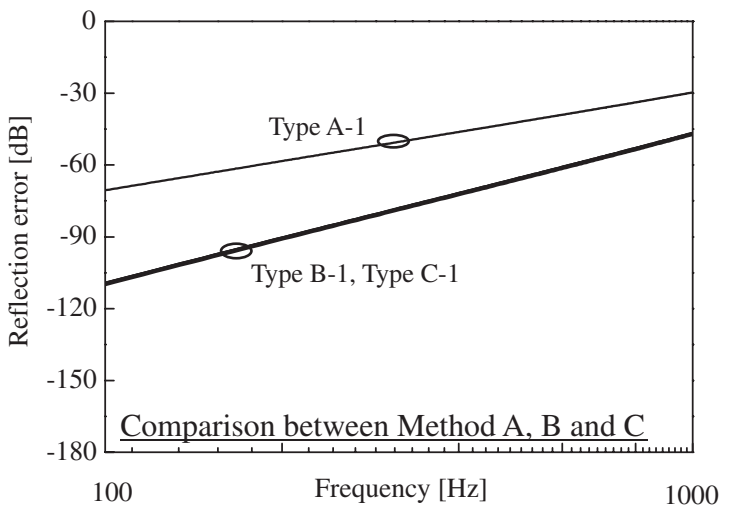

Fig. 8 Calculation results of reflection errors (Study 1).

$0.19 \mathrm{~cm}(=4 \mathrm{~cm} / 21)$. Iterative calculation was not performed and the time intervals, $\Delta t_{\text {fine }}$ and $\Delta t_{\text {coarse }}$, were set to be $5.0 \times 10^{-6} \mathrm{~s}$ in both conditions. The conditions are named Type B-1 (the numerical scheme is Method B, and the mesh ratio is 1:21), Type B-2 (Method $\mathrm{B}$ and 1:3), 


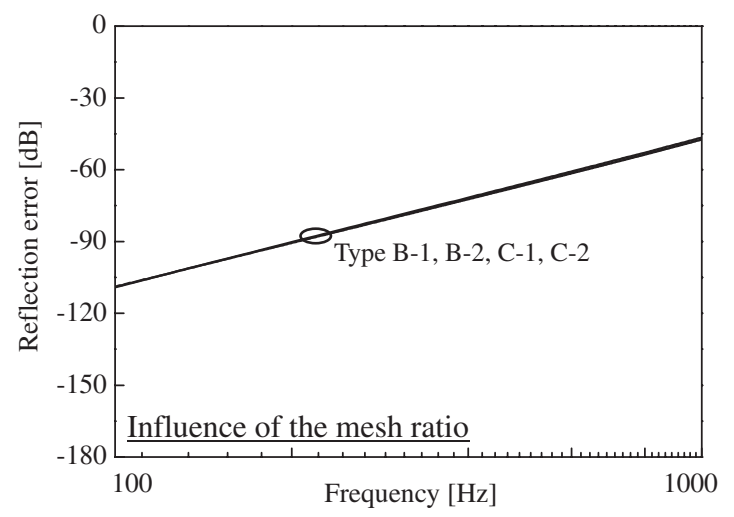

Fig. 9 Calculation results of reflection errors (Study 2).

Type C-1 (Method C and 1:21), Type C-2 (Method C and 1:3).

The calculated reflection errors are shown in Fig. 9. All types have almost the same values. It shows that the mesh ratio has no influence on the reflection error in Method B and $\mathrm{C}$.

Study 3: Influence of iteration number

The mesh ratio of 1:21 was adopted and the iteration number for the iterative calculation of the fine mesh area was varied as one (no iteration), 7 times and 21 times for Methods B and C. The coarse mesh size, $\Delta x_{\text {coarse }}$, was set to be $4 \mathrm{~cm}$ and the fine mesh size, $\Delta x_{\text {fine }}$, was $0.19 \mathrm{~cm}$ $(=4 \mathrm{~cm} / 21)$. The time interval, $\Delta t_{\text {fine }}$ was set to be $5.0 \times 10^{-6} \mathrm{~s}$, and $\Delta t_{\text {coarse }}$ for each situation was set to be $5.0 \times 10^{-6} \mathrm{~s}$ (no iteration), $3.5 \times 10^{-5} \mathrm{~s}\left(=5.0 \times 10^{-6} \mathrm{~s} \times\right.$ $7,7$ times $)$ and $1.05 \times 10^{-4} \mathrm{~s}\left(=5.0 \times 10^{-6} \mathrm{~s} \times 21,21\right.$ times), respectively. These conditions are named Types B-1-1, B-1-7, B-1-21 (Method B), C-1-1, C-1-7 and C-1-21 (Method C).

The calculated reflection errors are shown in Fig. 10. Whereas the reflection errors of Type B-1-7, Type B-1-21, Type C-1-7, and Type C-1-21 in the low-frequency range become higher than those of Type B-1-1 and C-1-1, the error in the high-frequency range of around $1 \mathrm{kHz}$ does not become very higher as the iteration number increases. This result indicates that the time interval, $\Delta t$, of the FDTD calculation for the simulated sound field discretized with a coarse mesh can be wider, and the time necessary for computation can be shortened. It contributes to such practical situations as sound insulation design phases of wall constructions.

According to the obtained results described above, lower reflection error can be obtained by using Methods B and $\mathrm{C}$ rather than Method A. Furthermore, from the viewpoint of computational cost, Method $\mathrm{C}$ is better than $\mathrm{B}$ when the mesh ratio between the coarse and fine mesh is the same between the two methods, because more calculation time for the particle velocity at the boundary is needed in Method B than in Method C.

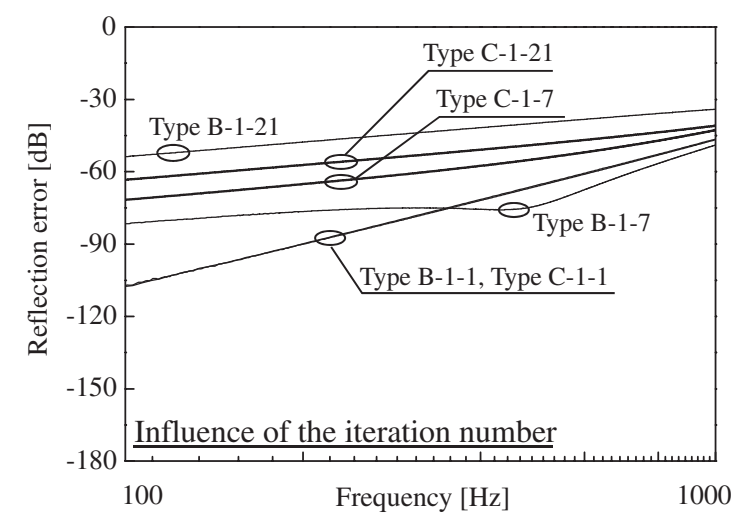

Fig. 10 Calculation results of reflection errors (Study 3).

(2) Study of 2-dimensional sound field

The reflection error is also investigated by FDTD analysis for the 2-dimensional sound field, as shown in Fig. 6. In this study, reflection error, which arises in the case of oblique incidence of sound waves into the boundary between coarse and fine mesh, is investigated. Transient responses at 5 receiving points shown in Fig. 6 were obtained by FDTD and the reflection error was basically calculated by the method described above. The procedure described below is performed additionally.

In order to correct the distance attenuation between the direct and the reflection sound, the following correction value, $L_{\text {correct }}$, was calculated and added to the reflection error.

$$
L_{\text {correct }}=10 \log \frac{r_{2}+r_{3}}{r_{1}}
$$

where $r_{1}$ is the direct path length from the source point to the receiving points, and $r_{2}$ and $r_{3}$ are reflection path lengths from the source to receiving points via a boundary surface (see Fig. 6).

The incident angle, $\theta$, shown in Fig. 6 is set as follows: $63.4^{\circ}(\mathrm{P} 1), 56.4^{\circ}(\mathrm{P} 2), 45.0^{\circ}(\mathrm{P} 3), 26.5^{\circ}(\mathrm{P} 4)$, and $0^{\circ}(\mathrm{P} 5)$. The conditions are named Type $\mathrm{P}-1$, Type $\mathrm{P}-2$, Type $\mathrm{P}-3$, Type P-4 and Type P-5.

In Fig. 6, the sound source with a Gaussian profile that includes the frequency components up to $1 \mathrm{kHz}$ was adopted. The sound field was surrounded by perfectly matched layers (PML) [18] in order to avoid sound reflection at the boundary. The mesh sizes, $\Delta x_{\text {coarse }}$ and $\Delta y_{\text {coarse }}$ are both set to be $4 \mathrm{~cm}$, and $\Delta x_{\text {fine }}$ and $\Delta y_{\text {fine }}$ are $1.33 \mathrm{~cm}(=4 \mathrm{~cm} / 3)$ and $4 \mathrm{~cm}$, respectively. The time intervals, $\Delta t_{\text {fine }}$ and $\Delta t_{\text {coarse }}$, were set to be $8.0 \times 10^{-6} \mathrm{~s}$, which fulfills the stability condition for the fine mesh area. As the numerical scheme for the calculation for the boundary, Method C was adopted.

The calculated reflection errors are shown in Fig. 11. As the receiving point is farther from the source point and the incident angle is larger, the reflection errors of Type 


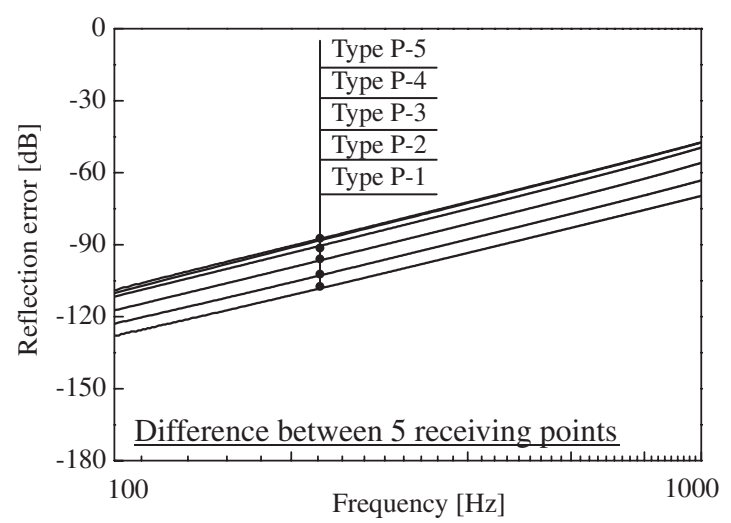

Fig. 11 Calculation results of reflection errors (2dimensional study).

P-1, Type P-2, Type P-3, and Type P-4 become lower than that of normal incidence, Type $\mathrm{P}-5$, in all frequency ranges. The reflection error arising at the boundary in the case of oblique incidence does not increase compared with the case of normal incidence. In the next chapter, Method C is applied to model a fine gap in the peripheral parts of a window sash and to calculate sound transmission characteristics through the gap.

\section{LEAK TRANSMISSION CHARACTERISTICS OF PERIPHERAL GAPS AROUND THE WINDOW SASH}

In order to predict leak transmission characteristics through narrow gaps in the peripheral parts of a window sash, 3-dimensinoal FDTD with the nonuniform-mesh system was applied. In this chapter, first, details of the laboratory experiment are described. Second, details of the numerical analysis using nonuniform-mesh FDTD is described. In the FDTD analysis, a pair of reverberation rooms and narrow gap in the separation wall between two rooms are numerically modeled, and sound transmission characteristics through the narrow gap are calculated. Lastly, the numerical results are compared with the measurement results and the applicability of the numerical method is discussed.

\subsection{Laboratory Experiment}

In this research, we referred the element normalized level difference, $D_{\mathrm{n}, \mathrm{e}}$, of each part of a window sash measured by Suda et al. [12]. In this measurement, the scanning intensity method is adopted to obtain the transmitting sound energy through each part of the window, separately. The measurement method is described hereafter.

The window sash shown in Fig. 12 is set in the separation wall between a reverberation room and an anechoic room (see Fig. 13). The dimensions of the

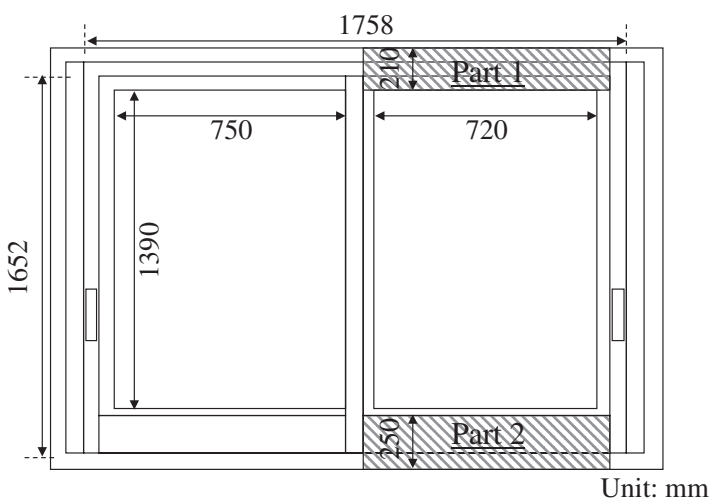

Fig. 12 Elevation of window sash under investigation.

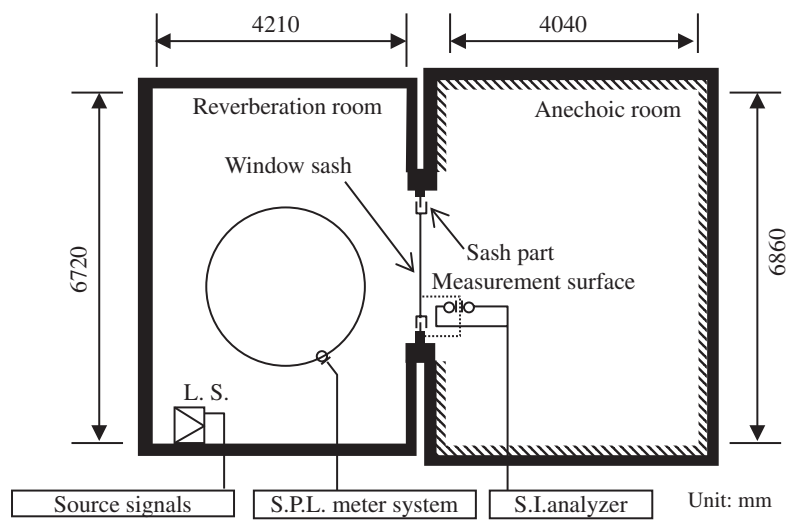

Fig. 13 A pair of a reverberation room and anechoic room.

measurement rooms are shown in Fig. 13. The transmitting sound power level, $L_{\mathrm{w}}$, from the upper and lower sashes, Part 1 and Part 2, were obtained using the scanning intensity method referring to ISO 15186-1 [19]. Then, averaged sound pressure level, $\bar{L}_{1}$, in the reverberation room was also obtained and the element normalized level difference, $D_{\mathrm{n}, \mathrm{e}}$, was calculated using

$$
D_{\mathrm{n}, \mathrm{e}}=\bar{L}_{1}-L_{\mathrm{W}}-6+10 \log _{10} S_{0},
$$

where $S_{0}$ is the reference area $\left(S_{0}=1.0 \mathrm{~m}^{2}\right)$.

\subsection{Numerical Analysis}

In the numerical analysis, the inner shape of the sash treated as described in Section 3.1 is numerically modeled and the sound energy transmitting through the narrow gap in the sash is calculated by FDTD. In this analysis, the sound insulation performance of the gap is predicted by modeling a pair of reverberation rooms and the gap of interest in the separation wall between the rooms. On this occasion, the use of the scanning intensity method, the same as in the measurement procedure, is desirable however, it is difficult to calculate sound intensity in a steady sound field by FDTD analysis. For this reason, the reverberation method was applied. Additionally, in [20], 


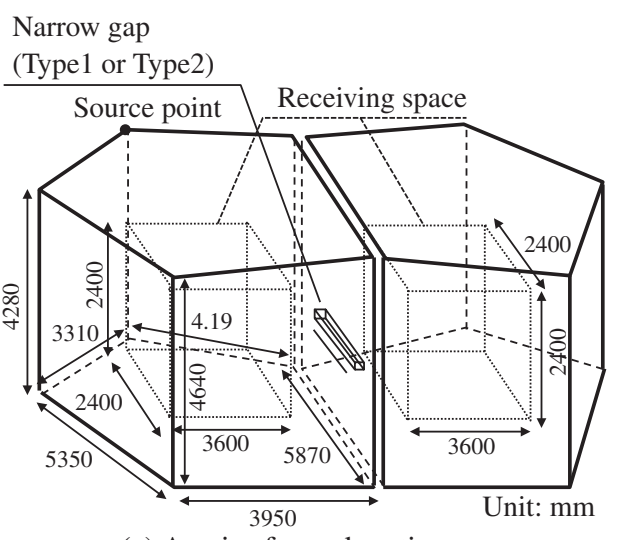

(a) A pair of reverberation rooms

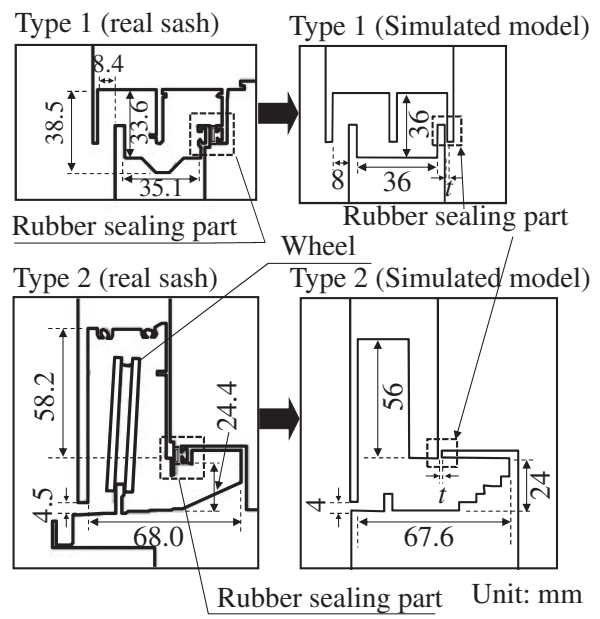

(b) Cross section of the modeled gaps

Fig. 14 A pair of reverberation rooms and modeled gaps.

values of sound transmission loss measured by both the sound intensity method and reverberation method were compared and found to agree well. Then, the numerical results were compared with the results of measurement obtained by the scanning intensity method.

A pair of irregularly shaped reverberation rooms and a narrow gap included in the separation wall between the two rooms were numerically modeled, as shown in Fig. 14(a), and the cross sections of the narrow gaps inside the aluminum sash were modeled as shown in Fig. 14(b). The gap existing in Part 1 (Fig. 12, upper side) is named Type 1 and that in Part 2 (Fig. 12, lower side) is named Type 2. Although the gaps surrounded by broken lines in Fig. 14(b) are sealed with rubber packing, leak transmission through the gap is not perfectly prevented because fairly narrow clearances partially exist between the aluminum frame of the sash part and the rubber packing. The narrow gap caused by the imperfect sealing is modeled as shown in the figure. The width of the clearance, $t$, is varied as $0.12 \mathrm{~mm}$ and $0.46 \mathrm{~mm}$ for each of Type 1 and Type 2. These clearances of $0.12 \mathrm{~mm}$ and $0.46 \mathrm{~mm}$ were obtained by setting an adequate mesh ratio of 1:(odd number) between fine and coarse meshes. The procedure of setting the arrangement of fine and coarse meshes for nonuniformmesh FDTD is described below.

The detailed mesh structures of the sound field around the gap (Type 1) are shown in Figs. 15(a), (b), and (c). First, the sound field inside the reverberation rooms is spatially discretized by Mesh (a) of $\Delta x=36 \mathrm{~mm}, \Delta y=$ $36 \mathrm{~mm}$ and $\Delta z=36 \mathrm{~mm}$. In order to model the shape of the finely shaped gap accurately, a mesh of $4 \mathrm{~mm} \times 4 \mathrm{~mm}$ is necessary. The mesh ratio between $4 \mathrm{~mm}$ and $36 \mathrm{~mm}$ is $1: 9$ and nonuniform-mesh FDTD can be applied. Then, the sound field near the gap is discretized by Mesh (b) of $\Delta x=4 \mathrm{~mm}, \Delta y=36 \mathrm{~mm}$ and $\Delta z=4 \mathrm{~mm}$ as shown in Fig. 15(a). Next, in order to model the clearance in the rubber sealing part (clearance: $0.12 \mathrm{~mm}$ ), a mesh of $\Delta x=0.12 \mathrm{~mm}, \Delta y=36 \mathrm{~mm}$ and $\Delta z=4 \mathrm{~mm}$ is necessary (Mesh (c)). In this mesh system, the mesh ratio of 1:33, for the fine mesh, is adopted.

When the clearance of the rubber sealing part is $0.46 \mathrm{~mm}$, the sound field is discretized by Mesh (d): $\Delta x=$ $0.23 \mathrm{~mm}, \Delta y=36 \mathrm{~mm}$ and $\Delta z=4 \mathrm{~mm}$ (the mesh ratio between $0.23 \mathrm{~mm}$ and $4 \mathrm{~mm}$ is $1: 17$ ).

In the case of the lower side gap, Type 2, the sound field near the rubber sealing part is also discretized by a mesh of the same size as that for the upper side gap (Fig. 16). The wheels that support the window sash is not modeled in this calculation.

Three-dimensional FDTD analysis was performed using the high-order scheme with 8 reference points in order to avoid phase error [16]. The time interval adopted in this analysis is $8.0 \times 10^{-6} \mathrm{~s}$. For the rubber sealing parts, iterative calculation was performed 30 times was performed and the time interval was locally set as $2.7 \times 10^{-7} \mathrm{~s}$ $\left(=8.0 \times 10^{-6} / 30\right)$. As shown in Fig. 14(a), the sound source was set in a corner of the room by supplying the initial sound pressure distribution with a Gaussian profile that includes frequency components up to $2 \mathrm{kHz}$. The frequency components of the calculated impulse response ranged from $89 \mathrm{~Hz}$ to $1.795 \mathrm{kHz}$, which includes $1 / 3$ Oct. bands from $100 \mathrm{~Hz}$ to $1.6 \mathrm{kHz}$, were used for calculating the element normalized level difference, $D_{\mathrm{n}, \mathrm{e}}$. The upper limit frequency of $1.795 \mathrm{kHz}$ is determined in consideration of the spatial mesh size and the adopted FDTD scheme referring to the research [16]. The normal incidence absorption coefficients of surfaces of reverberation rooms are set to be 0.026 by assigning a normal acoustic impedance of $62,000 \mathrm{Ns} / \mathrm{m}^{3}$ for all boundaries. In this case, the statistical incidence absorption coefficient is calculated to be 0.05 using Paris's theory [21], and the reverberation time of the reverberation room is estimated to be $2.95 \mathrm{~s}$ by substituting the statistical incidence absorption coefficient into Sabine's Equation [22]. On such a setting, the duration of FDTD calculation is set to be 3 s (375,000 time steps). 

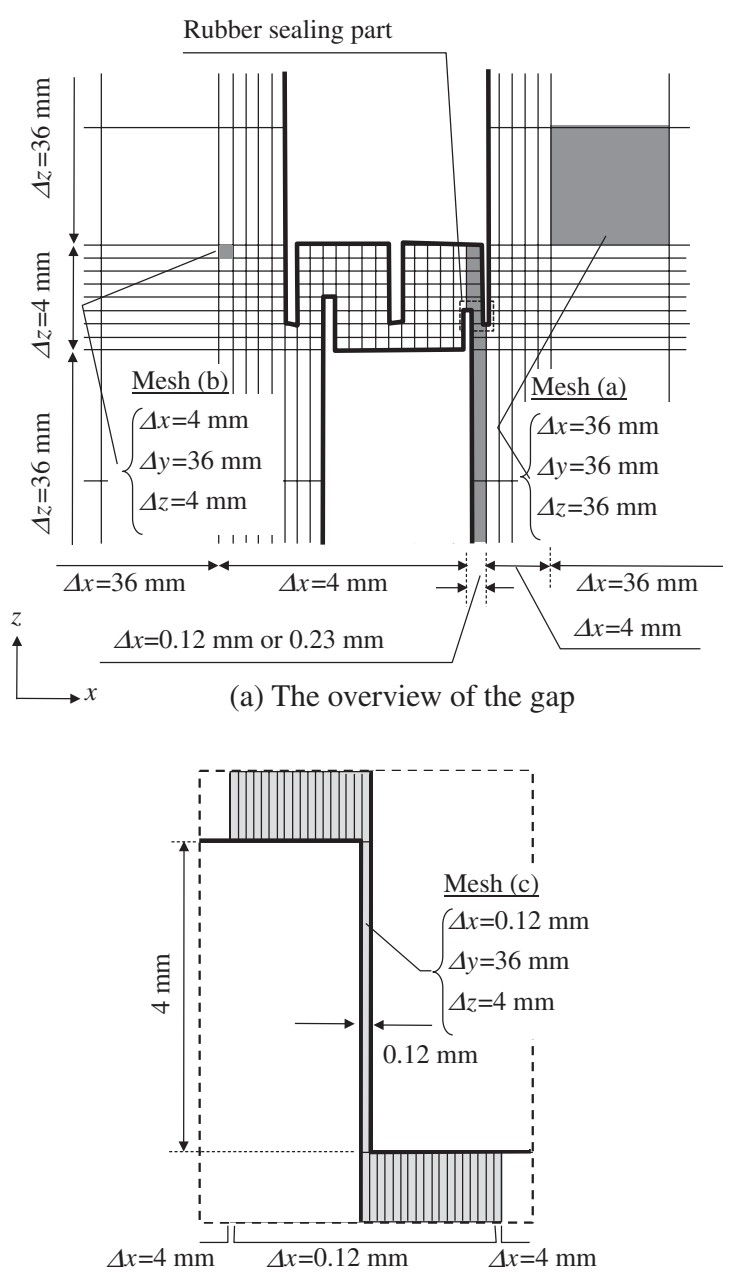

(b) Expanded view of the rubber sealing part (Clearance: $0.12 \mathrm{~mm}$ )

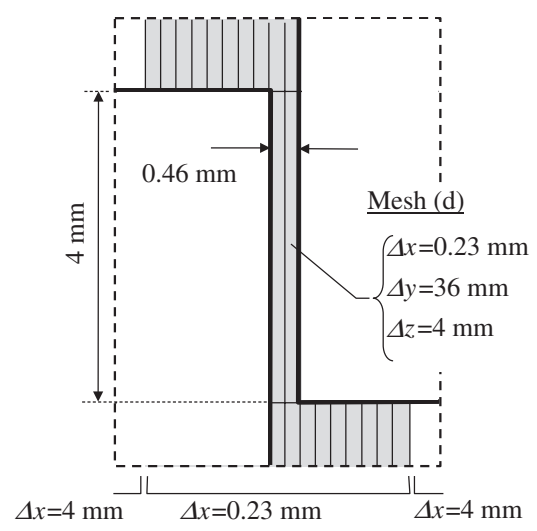

(c) Expanded view of the rubber sealing part (Clearance: $0.46 \mathrm{~mm}$ )

Fig. 15 Detailed FDTD mesh structures in the case of Type 1.

In the source and receiving rooms, 490 receiving points were distributed in the receiving spaces $(2.4 \mathrm{~m} \times 2.4 \mathrm{~m} \times$ $3.6 \mathrm{~m}$ ) indicated by dotted line in Fig. 14(a). Referring to ISO [23], the receiving space is set at least $0.7 \mathrm{~m}$ from the boundary surface, the gap opening, and $1.0 \mathrm{~m}$ away from the sound source point inside the source room. Separating distances between the adjacent receiving points are $0.4 \mathrm{~m}$.

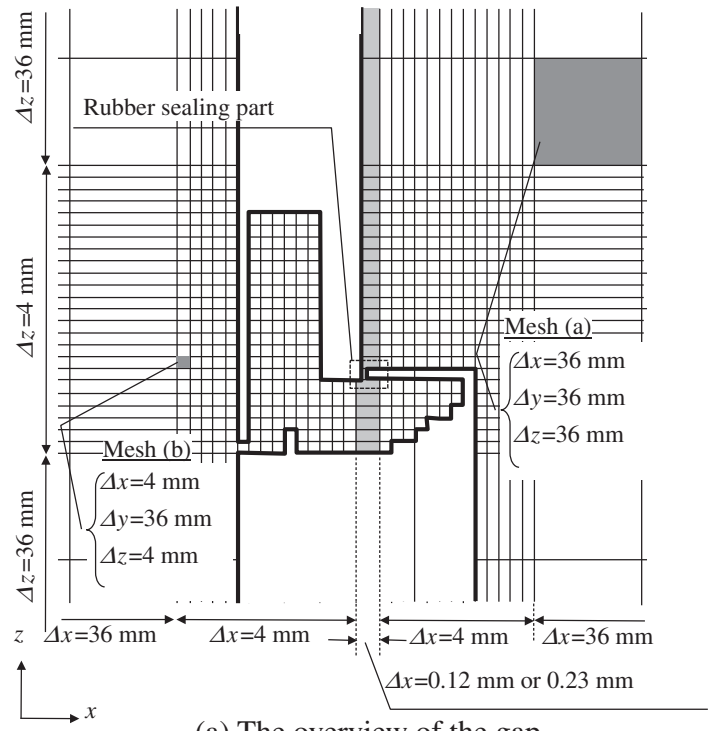

(a) The overview of the gap

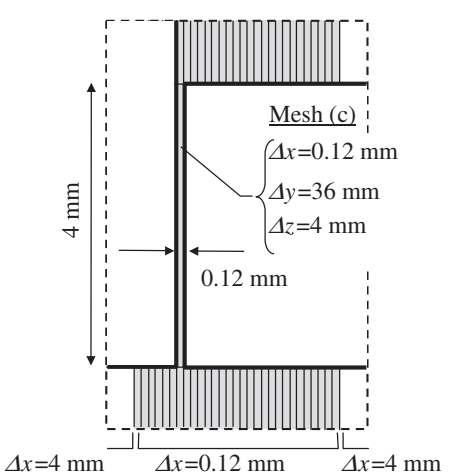

(b) Expanded view of the rubber sealing part (Clearance: $0.12 \mathrm{~mm}$ )

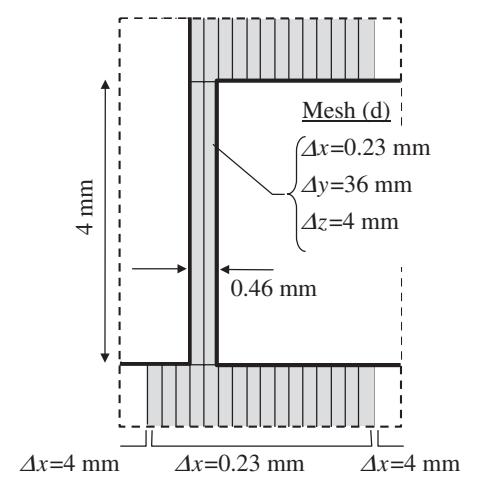

(c) Expanded view of the rubber sealing part (Clearance: $0.46 \mathrm{~mm}$ )

Fig. 16 Detailed FDTD mesh structures in the case of Type 2.

Then, element normalized level difference, $D_{\mathrm{n}, \mathrm{e}}$, is calculated by following equation.

$$
D_{\mathrm{n}, \mathrm{e}}=\bar{L}_{1}-\bar{L}_{2}+10 \log _{10} \frac{S_{0}}{A_{2}},
$$

where $\bar{L}_{1}$ and $\bar{L}_{2}$ are the averaged sound energy levels in the source and the receiving rooms, $S_{0}$ is the reference area $\left(S_{0}=1.0 \mathrm{~m}^{2}\right), A_{2}$ is the equivalent sound absorption area 

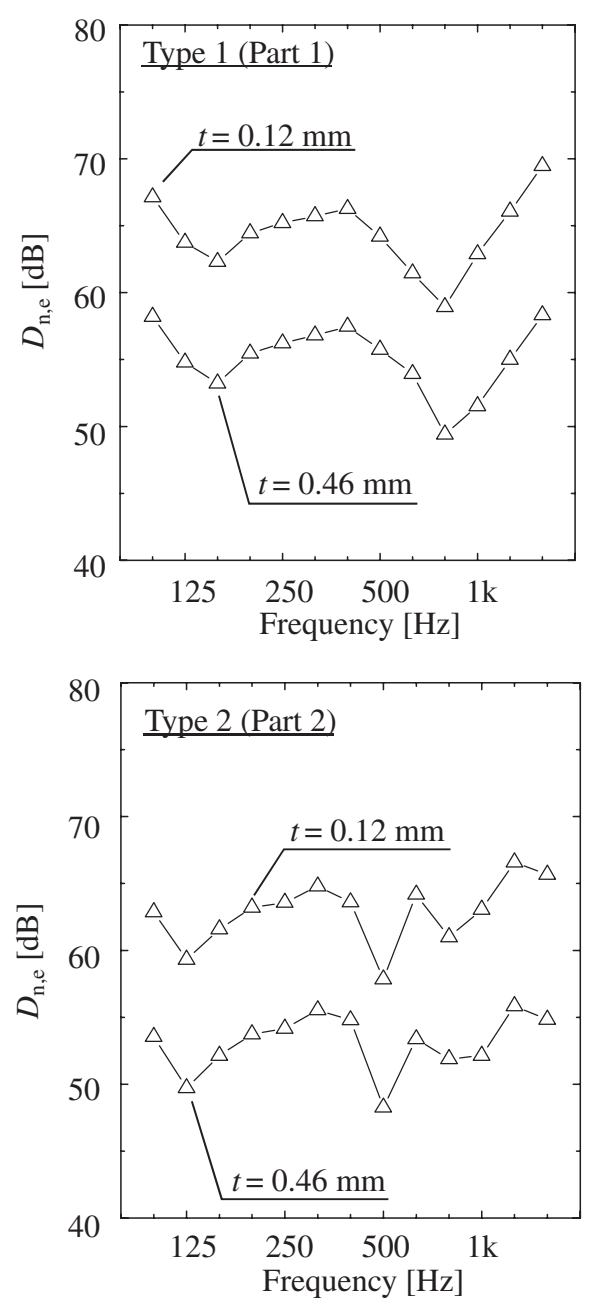

Fig. 17 Calculation results of element normalized level.

$\left(A_{2}=55.3 V_{2} / c T_{2}\right)$ of the receiving room, and $V_{2}$ and $T_{2}$ are the volume $\left(164 \mathrm{~m}^{3}\right)$ and the reverberation time of the receiving room, respectively. The reverberation time in each $1 / 3$ Oct. band is calculated by the integrated impulse response method.

\subsection{Result}

Figure 17 shows the calculation results. In Type 1 under the condition of $t=0.46 \mathrm{~mm}$, a dip is observed at $800 \mathrm{~Hz}$. This dip is considered to be a sound insulation defect caused by Helmholtz resonance of the air volume inside the sash. $D_{\mathrm{n}, \mathrm{e}}$ under the condition of $t=0.12 \mathrm{~mm}$ is about $10 \mathrm{~dB}$ higher than that under the condition of $t=0.46 \mathrm{~mm}$ in all frequency bands.

In Type 2, the dip frequency is $500 \mathrm{~Hz}$ and it is lower than that of Type $1(800 \mathrm{~Hz})$. It is because the volume of air inside the gap of Type 2 is larger than that in the case of Type 1. $D_{\mathrm{n}, \mathrm{e}}$ under the condition of $t=0.46 \mathrm{~mm}$ is also lower than that under the condition of $t=0.12 \mathrm{~mm}$ by about $10 \mathrm{~dB}$ in all frequency bands. From these results, it is indicated that the sound insulation performance of the gap
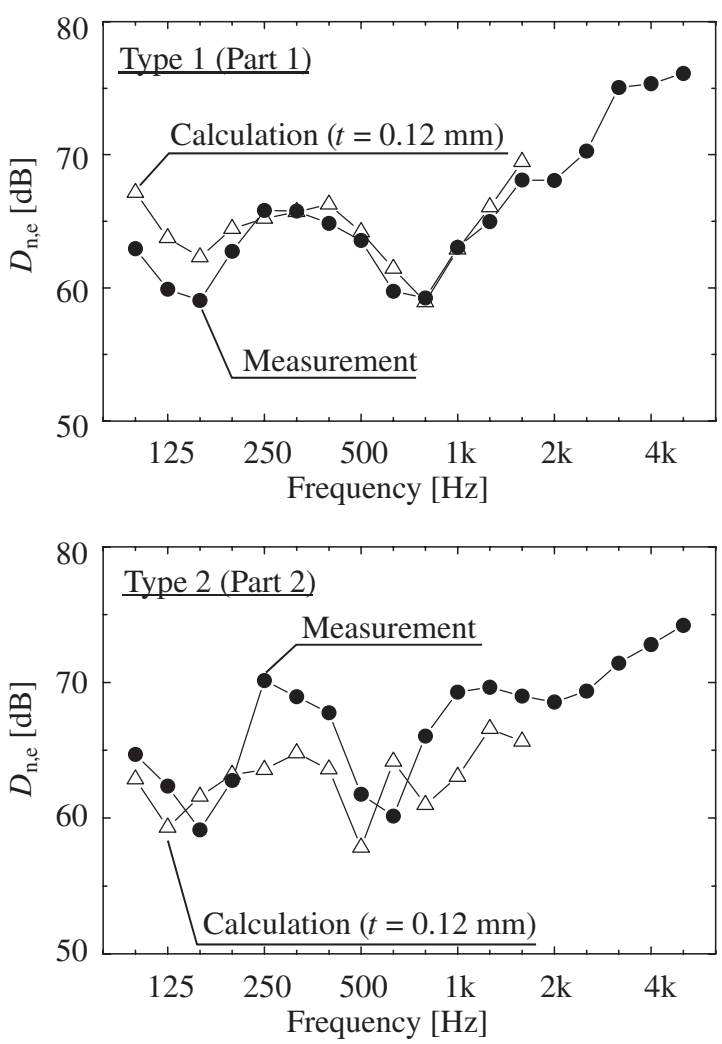

Fig. 18 Calculation and measurement results of element normalized level difference, $D_{\mathrm{n}, \mathrm{e}}$.

is degraded when the clearance of the rubber sealing part is slightly widened.

Figure 18 shows the calculation and measurement results. As a calculation result, $D_{\mathrm{n}, \mathrm{e}}$ under the condition of $t=0.12 \mathrm{~mm}$ is in good agreement with the measurement results. Under the condition of Type 1, frequency characteristics of measurement and calculation results are in fairly good agreement. On the other hand, for Type 2, the dip frequency of the calculation result in the $500 \mathrm{~Hz}$ band does not agree with that of the measurement result, whereas tendencies of the frequency characteristics of the measurement and calculation results agree well. In this analysis, the complex structure of a wheel that is set inside the lower gap is not modeled and the volume of air was overestimated by neglecting the volume of the wheel structure. As a result, the dip frequency (resonant frequency of the Helmholtz resonance) in the calculation result was observed in the lower frequency band than that of the measurement result.

\section{CONCLUSIONS}

Leak transmission characteristics through narrow gaps of small dimensions were investigated by numerical analysis. The narrow gaps in the sash of a window were modeled and their leak transmission characteristics were calculated by the FDTD method using a nonuniform-mesh system. The leak transmission characteristics through these 
gaps measured by the scanning intensity method were compared with the calculation results. A good agreement between the frequency characteristics of the sound insulation obtained by measurement and calculation was confirmed.

The authors are also investigating a prediction method of the sound insulation performance of plates, such as glass plates and plasterboards, by vibro-acoustic FDTD analysis [10]. The nonuniform-mesh FDTD and vibro-acoustic FDTD are combined and the development of a prediction method for sound insulation of a complex wall system, such as a window sash, should be investigated in the future.

\section{REFERENCES}

[1] F. Ingerslev and A. K. Nilsson, "On the transmission of sound through small apertures and narrow slits," Acoust. Lab. Acad. Tech. Sci., Copenhagen, Publication No. 1 (1944).

[2] H. Levine and J. Schwinger, "On the theory of diffraction by an aperture in an infinite plane screen," Phys. Rev., 74, 958974 (1948).

[3] Y. Nomura and S. Inawashiro, "On the transmission of acoustic waves through a circular channel of a thick wall," Sci. Rep. Tohoku Univ. (1), 12B, 57-71 (1960).

[4] M. C. Gomperts, "The "sound insulation" of circular and slitshaped aperture," Acustica, 14, 1-16 (1964).

[5] G. P. Wilson and W. W. Soroka, "Approximation to the diffraction of sound by a circular aperture in a rigid wall of finite thickness," J. Acoust. Soc. Am., 37, 286-297 (1965).

[6] P. T. Lewis, "Effect of frame construction on the sound insulation of unsealed windows," Appl. Acoust., 12, 15-24 (1979).

[7] M. A. Burgess, "Resonator effects in window frames," J. Sound Vib., 103, 323-332 (1985).

[8] V. Hongisto, "Sound insulation of doors - part 1: prediction models for structural and leak transmission," J. Sound Vib., 230, 133-148 (2000).

[9] V. Hongisto, J. Keränen and M. Lindgren, "Sound insulation of doors - part 2: comparison between measurement results and predictions," J. Sound Vib., 230, 149-170 (2000).

[10] T. Asakura and S. Sakamoto, "Finite-difference time-domain analysis of sound insulation performance of wall systems," Build. Acoust., 16, 267-281 (2009).

[11] H. Jiang and H. Arai, "Analysis of computation error in antenna's simulation by using non-uniform mesh FDTD," IEICE Trans. Commun., E83-B, 1544-1553 (2000).

[12] N. Suda, F. Satoh, H. Yano, A. Sunaga, T. Yokota, S. Sakamoto and H. Tachibana, "Analysis of partial sound insulation performance of a sash by sound intensity method," Proc. Spring Meet. INCE/J., pp. 109-112 (2003) (in Japanese).

[13] Y. Maeda, S. Yamamoto, D. Ushirogouchi, K. Asanuma and T. Maeda, "A Study on the application conditions of FDTD subgridding algorithm for the analysis of Yagi-Uda antennas placed in the vicinity of building structures," IEICE Trans. Antennas Propag., 106(491), No. 7, pp. 49-54 (2007) (in Japanese).

[14] K. Okubo, Y. Yoshida, T. Tsuchiya, N. Takeuchi and N. Tagawa, "Numerical dispersion in analysis for electromagnetic wave propagation using asymmetric finite difference time domain (A-FDTD) method with higher order FDTD," IEICE Trans. Commun., 107, No. 210, AP2007-84, 61-65 (2007) (in Japanese).

[15] T. Uno, Finite Difference Time Domain Method for Electro- magnetic Field and Antennas (Corona Publishing Co., Ltd., Tokyo, 2003) (in Japanese).

[16] S. Sakamoto, "Phase-error analysis of high-order finite difference time domain scheme and its influence on calculation results of impulse response in closed sound field," Acoust. Sci. \& Tech., 28, 295-309 (2007).

[17] T. Yokota, S. Sakamoto and H. Tachibana, "Sound field simulation method by combining finite difference time domain calculation and multi-channel reproduction technique," Acoust. Sci. \& Tech., 25, 15-23 (2004).

[18] J. P. Berenger, "A perfectly matched layer for the absorption of electromagnetic waves," J. Comput. Phys., 114, 185-200 (1994).

[19] ISO 15186-1:2000, Acoustics - Measurement of sound insulation in buildings and of building elements-, Part 3: Laboratory measurements of airborne sound insulation of building elements (2000).

[20] J. C. S. Lai, M. A. Burgess, P. P. Narang and K. Mikl, "Transmission loss measurements: comparisons between sound intensity and conventional methods," Proc. INTERNOISE 91, pp. 1029-1032 (1991).

[21] E. T. Paris, "On the coefficient of sound-absorption measured by the reverberation method," Philos. Mag., 5, 489-497 (1928).

[22] W. C. Sabine, Collected Papers on Acoustics (Harvard University Press, Cambridge, Mass., 1922).

[23] ISO 140-3:1995, Acoustics - Measurement of sound insulation in buildings and of building elements using sound intensity —, Part 1: In-situ condition (1995).

\section{Appendix}

The calculation method of the sound pressure gradient at the boundary between fine and coarse meshes is described in this appendix. In Fig. A.1, the sound pressures, $p_{1}, p_{2}, p_{3}$, and $p_{4}$ at the points, $x_{1}, x_{2}, x_{3}$, and $x_{4}$ are known. The following equations (A-2), (A.3), (A.4), and (A.5), are satisfied assuming that a polynomial function, Eq. (A.1), includes the four points, $x_{1}, x_{2}, x_{3}$, and $x_{4}$.

$$
\begin{aligned}
& y=a x^{3}+b x^{2}+c x+d \\
& a x_{1}^{3}+b x_{1}^{2}+c x_{1}+d=p_{1} \\
& a x_{2}^{3}+b x_{2}^{2}+c x_{2}+d=p_{2} \\
& a x_{3}^{3}+b x_{3}^{2}+c x_{3}+d=p_{3} \\
& a x_{4}^{3}+b x_{4}^{2}+c x_{4}+d=p_{4}
\end{aligned}
$$

The linear equations (A.2), (A.3), (A.4), and (A.5) are described as follows.

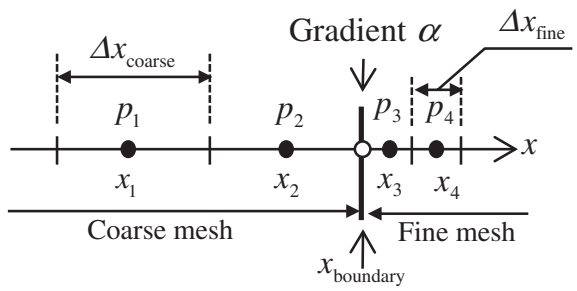

Fig. A.1 One-dimensional sound field around the boundary between coarse and fine meshes. 


$$
\left[\begin{array}{rrrr}
x_{1}^{3} & x_{1}^{2} & x_{1}^{1} & 1 \\
x_{2}^{3} & x_{2}^{2} & x_{2}^{3} & 1 \\
x_{3}^{3} & x_{3}^{2} & x_{3}^{1} & 1 \\
x_{4}^{3} & x_{4}^{2} & x_{4}^{1} & 1
\end{array}\right]\left[\begin{array}{l}
a \\
b \\
c \\
d
\end{array}\right]=\left[\begin{array}{l}
p_{1} \\
p_{2} \\
p_{3} \\
p_{4}
\end{array}\right]
$$

When the origin of the coordinate on the $x$ axis is set at $x_{\text {boundary, }}$ linear equation (A.6) is transformed into the following equation.

$$
\left[\begin{array}{cccc}
\left(-1.5 \cdot \Delta x_{\text {coarse }}\right)^{3} & \left(-1.5 \cdot \Delta x_{\text {coarse }}\right)^{2} & -1.5 \cdot \Delta x_{\text {coarse }} & 1 \\
\left(-0.5 \cdot \Delta x_{\text {coarse }}\right)^{3} & \left(-0.5 \cdot \Delta x_{\text {coarse }}\right)^{2} & -0.5 \cdot \Delta x_{\text {coarse }} & 1 \\
\left(0.5 \cdot \Delta x_{\text {fine }}\right)^{3} & \left(0.5 \cdot \Delta x_{\text {fine }}\right)^{2} & 0.5 \cdot \Delta x_{\text {fine }} & 1 \\
\left(1.5 \cdot \Delta x_{\text {fine }}\right)^{3} & \left(1.5 \cdot \Delta x_{\text {fine }}\right)^{2} & 1.5 \cdot \Delta x_{\text {fine }} & 1
\end{array}\right]\left[\begin{array}{l}
a \\
b \\
c \\
d
\end{array}\right]=\left[\begin{array}{l}
p_{1} \\
p_{2} \\
p_{3} \\
p_{4}
\end{array}\right]
$$

The coefficients, $a, b, c$, and $d$ are obtained by solving Eq. (A.7).

The gradient, $\alpha$, at the point, $x_{\text {boundary }}$, (Fig. 1) is calculated by the following equation, which was obtained by differentiating Eq. (A.1) with respect to $x$ and substituting $x_{\text {boundary }}$ for $x$.

$$
\alpha=3 a x_{\text {boundary }}^{2}+2 b x_{\text {boundary }}+c
$$

Then, the gradient, $\alpha$, is described by the following equation, which was obtained by substituting 0 for $x_{\text {boundary }}$ in Eq. (A.8)

$$
\alpha=c
$$

